\title{
Adaptación de la asignatura de Catalogación Descriptiva al Espacio Europeo de Educación Superior (EEES)
}

Adaptation of the "descriptive cataloguing" subject to the European Higher Education Area

Yolanda Martín González (1) y Ana Belén Ríos Hilario (2)

(1) Dpto. de Biblioteconomía y Documentación (Universidad de Salamanca), C/ Francisco de Vitoria 6-16, 37008 Salamanca, ymargon@usal.es, (2) anarihi@usal.es

\section{Resumen}

Se describe el proceso de adaptación de la asignatura de Catalogación Descriptiva a las directrices marcadas por el Espacio Europeo de Educación Superior (EEES). Tras indicar los puntos claves que estipula dicho modelo, se detalla la inserción de la materia de Catalogación dentro de los planes de estudios de la Universidad de Salamanca. Seguidamente, se definen los ejes fundamentales que hay que tener en cuenta en el diseño de la asignatura y, mediante una serie de tablas, se muestra como se va configurando la misma tras llevar a la práctica tales pautas. Se concluye diciendo que el cambio de paradigma marcado por el EEES incide directamente en el alumno quien se convierte así en parte activa de dicho proceso.

Palabras clave: Espacio Europeo de Educación Superior (EEES). Catalogación descriptiva. Métodos de enseñanza.

"Debemos contar con una voluntad clara y convencida por parte del profesor [...] Esta voluntad nos llevará a conseguir la capacidad para hacerlo y a demandar a la universidad aquellos recursos y espacios de trabajos necesarios para poder llevarlo a cabo".

(Bernal Aguado)

\section{Introducción}

La Universidad española se encuentra inmersa en un proceso de gran responsabilidad como es "afrontar los cambios que supone el establecimiento del Espacio Europeo de Educación Superior (EEES) en pos de la integración europea, tal y como establece la Declaración de Bolonia (1999) cuyos objetivos deben lograrse en la fecha límite del año 2010" (Pinto Molina, 2006).

En estos últimos años los docentes universitarios hemos estado tomando conciencia de los cambios que conlleva este nuevo entorno, en el que el alumno se convierte en el eje fundamental del aprendizaje. Partiendo de esta premisa el

\begin{abstract}
The adaptation process of the subject of Descriptive Cataloguing to the guidelines marked by the European Higher Education Area (EHEA) is described. After indicating the key points stipulated in this model, the insertion of the matter of Cataloguing within the curricula of the University of Salamanca is detailed. Next, the fundamental axes that must be considered in the design of the subject are defined and its configuration is showed a series of tables. In conclusion, the shift of paradigm marked by the EEES affects the student directly, who becomes thus an active part of this process.
\end{abstract}

Keywords: European Higher Education Area. Cataloguing. Teaching Methods.

profesor tiene que cambiar su concepción en cuanto los métodos de enseñanza, entendiendo por éstos las distintas maneras de organizar y llevar a cabo los procesos de enseñanzaaprendizaje (clases teóricas, seminarios, talleres, tutorías, trabajo en grupo...). Sin embargo, una vez aprendida la teoría, su aplicación práctica no es una tarea fácil, de tal modo que el objetivo de este artículo es ofrecer una guía de las pautas que hemos seguido a la hora de adaptar la asignatura de Catalogación Descriptiva a las nuevas demandas que postula el EEES.

De tal manera que, una vez indicadas las claves sobre las que se sustenta este modelo de enseñanza, procedimos en primer lugar a realizar un recorrido por la situación que dicha asignatura ha tenido dentro de los planes de estudios de la Universidad de Salamanca. Posteriormente, y trás establecer las diferencias entre los planes actuales y los europeos, se presenta el esque- 
ma que debe seguirse a la hora de planificar las materias. Para ello, previamente definimos los ejes fundamentales que hay que tener en cuenta en el diseño de la asignatura y a continuación, y mediante una serie de tablas, mostramos como se va configurando nuestra asignatura tras llevar a la práctica tales premisas. Se concluye nombrando las principales diferencias que existen entra los actuales planes y los planes europeos.

\section{Las claves del EEES}

La primera pregunta que todos nos hacemos cuando escuchamos la denominación de Espacio Europeo de Educación Superior (EEES) es precisamente qué se esconde bajo este término, o en concreto, qué significa o qué comprende este nuevo paradigma de enseñanza universitaria. Acudiendo a los teóricos sobre la materia debemos entender este concepto "como una oportunidad para mejorar, para cambiar la universidad en algunos de sus lastres y modelos anquilosados de transmitir conocimientos científicos y formar investigadores en todos los campos del saber" (Hernández Díaz, 2006).

En relación con la definición, podemos enumerar cuáles son los motivos que nos llevan a la creación de este concepto: (1) incremento del número de titulados universitarios en Europa; (2) facilitar la eliminación de las barreras a la libre circulación de estudiantes y titulados en la EU y el resto de Europa; (3) hacer de Europa un lugar más atractivo para estudiar e investigar; (4)cCambio de paradigma educativo, es decir, se produce el paso de la enseñanza al aprendizaje autónomo; y (5) vincular la educación superior al aprendizaje a lo largo de toda la vida.

\subsection{Elementos claves en la adaptación al EEES}

Este nuevo EEES va a contar con una serie de elementos claves que lo diferencia del actual modelo de enseñanza y entre los que podemos citar los siguientes: (1) sistema de formación en dos niveles: grado y postgrado; (2) crédito ECTS; (3) suplemento europeo al título; (4) movilidad de estudiantes, PDI y PAS; (5) sistemas de garantía de calidad; (6) dimensión europea de la educación superior; y (7) aprendizaje a lo largo de toda la vida.

De entre todos ellos, destaca por su complejidad el crédito ECTS, por el que se entiende la "unidad de medida del trabajo realizado por el estudiante para cumplir los objetivos del programa de estudios oficial correspondiente". De tal forma que el número mínimo de horas por crédito será de 25 y el máximo de 30 . En un crédito ECTS se contabilizan no sólo las horas correspondientes a clases lectivas, teóricas o prácticas; sino también las horas de estudio; las dedicadas a la realización de seminarios, trabajos, prácticas o proyectos; y las exigidas para la preparación y realización de los exámenes y pruebas de evaluación. De tal forma que se recomienda que un curso académico conste de 60 créditos ECTS, repartidos entre un mínimo de 36 semanas y un máximo de 40 . Asimismo, la correcta aplicación de esta nueva unidad debe de traer consigo desde variados sistemas de evaluación, a una revisión y, en su caso, replanteamiento del material y metodologías docentes utilizadas hasta el momento; y aquí es precisamente donde entra en juego, si no un nuevo concepto, si una nueva comprensión y dimensión del mismo: la Guía docente (SPF, 2005).

Este instrumento, como planificación docente que es, representa una presentación básica de los principios que regirán el desarrollo de la asignatura o módulo. Para el profesor significa estructurar en "un mismo paquete": (a) una selección y ordenación de los contenidos académicos, y (b) una oferta didáctica, esto es, cómo van a ser trabajados esos contenidos y cómo van a ser evaluados los estudiantes.

Es función de la Guía docente, en primer lugar, ser un documento donde se concreta la oferta docente referida a una asignatura: es decir, contenidos, objetivos, formas de enseñanza, criterios de evaluación. Por otro lado, deber ser un instrumento al servicio del estudiante, el cual debe ofrecerle los elementos informativos suficientes para determinar qué es lo que se pretenda que aprenda, cómo se va a llevar a cabo el aprendizaje, bajo que condiciones y de que modo va a ser evaluado. En tercer lugar, el programa representa el compromiso del profesor entorno a diferentes criterios (contenidos, formas de trabajo, evaluación...) sobre los que irá desarrollando su enseñanza. Por último, debe ser un instrumento de transparencia, fácilmente comprensible y comparable entre las diferentes universidades en el marco del EEES.

Íntimamente ligado a las funciones que debe cumplir este instrumento, está precisamente la tarea de diseñar la Guía. Podemos decir que en toda Guía docente se tendrían que especificar los siguientes epígrafes: (1) datos de identificación, (2) introducción a la asignatura, (3) volumen de trabajo (4) objetivos generales, (5) contenidos mínimos (6) destrezas a adquirir, (7) habilidades sociales, (8) temario, (9) bibliografía de referencia, (10) conocimientos previos, (11) metodología, y (12) evaluación del aprendizaje. 
Respecto a las actuales Guías académicas nos llama la atención - no tanto en su denominación, sino en cuanto su definición- determinado puntos que pasaremos a comentar de modo más detallado a continuación. Es significativo el apartado Destrezas a adquirir, en el que deben definirse los procedimientos y métodos considerados como claves en el dominio de la asignatura y en el ejercicio de su aplicación práctica. Otro aspecto importante sería el de las Habilidades sociales, que engloba el conjunto de procedimientos y también de actitudes que se relacionan con la forma en la que el estudiante interactúa con su entorno social, tanto desde un punto de vista general - social y académicocomo desde una perspectiva centrada en el desarrollo futuro. En el apartado de Metodología deberá describirse la distribución y estructura de las horas teóricas, prácticas y de ordenador o laboratorio, en su caso. Finalmente, la Evaluación del aprendizaje debe explicitar los procedimientos de recogida de información para la evaluación, como por ejemplo, trabajos, informes, exámenes, así como los criterios que se utilizan para derivar la calificación.

\section{La catalogación y su adaptación al EEES}

\subsection{La asignatura de catalogación dentro de} los planes de estudio de la Diplomatura en ByD

La materia de catalogación ha estado presente en todos los planes de estudio desde la implementación de los estudios de Biblioteconomía y Documentación (ByD) en España allá por el año 1983 (Ubieto Artur, 1993).

La Universidad de Salamanca (USAL) en 1987 crea la Escuela Universitaria de Biblioteconomía y Documentación (Orden de 2 de octubre, B. O. E. del 20) y en ese mismo año comienza a funcionar la Diplomatura en Biblioteconomía y Documentación. En los primeros planes de estudios, la materia de catalogación se incluye dentro de la asignatura de Análisis documental, que se impartía en el primer curso de tal titulación, siendo anual y de carácter obligatorio.

En el año 1992 se crea la actual Facultad de Traducción y Documentación (R. D. 1050/92 de 31 de julio, B. O. E. de 26 de agosto), dado que se incorporaron los estudios conducentes a la Licenciatura en Traducción e Interpretación.

En 1993 se modificó el plan de estudios de la Diplomatura en Biblioteconomía y Documentación (Resolución de 17 de noviembre, B. O. E. de 2 de diciembre), apareciendo en el curso 9596 los primeros titulados con este plan. En estos planes, comienza a impartirse la asignatura de
Catalogación Descriptiva I: impresos modernos cuyas características son: materia troncal, con 4 créditos, a impartirse en un cuatrimestre y que los alumnos debían cursar en el segundo año de la diplomatura. Relacionadas con esta materia, se imparten las siguientes asignaturas: Análisis estructural del catálogo y Control de autoridades (troncales) y Catalogación descriptiva II: materiales especiales (optativa).

La oferta de titulaciones se amplia en 1994 con la Licenciatura en Documentación (Resolución de 30 de mayo, B. O. E. de 20 de junio). En los primeros planes de estudios la única asignatura relacionada de forma indirecta con la catalogación era la Gestión y evaluación de catálogos automatizados.

Los actuales planes de estudio datan del año 2000 (Resolución de 3 de octubre) en los que la asignatura de Catalogación sigue siendo troncal y continúa ofertándose en el segundo curso de la diplomatura, pero pasa a ser anual al tener 10 créditos. Las asignaturas que complementan la formación catalogadora son las siguientes: Introducción al análisis y recuperación de la información y Organización de catálogos en línea (obligatorias) y Catalogación descriptiva II: materiales especiales (optativa).

En la licenciatura se ofertan las siguientes asignaturas: una directamente relacionada, Descripción y organización de documentos digitales; y otra que tiene que ver no tanto con el proceso - la catalogación- sino más bien con su producto -el catálogo-, Evaluación de catálogos en línea.

Este recorrido por los planes de estudio nos lleva al año 2006, en el que el Departamento de Biblioteconomía y Documentación de la USAL decide poner como proyectos pilotos de adaptación al EEES las asignaturas de Archivística y Catalogación descriptiva I: impresos modernos ambas del segundo curso de la Diplomatura.

\subsection{El cambio de los planes actuales} a los planes europeos

La adaptación al EEES debe hacerse desde dos puntos de vista. En primer lugar, debe producirse un cambio en la metodología, dónde el alumno se convierte en el eje esencial de la educación; en segundo lugar, se debe producir un cambio tecnológico, en el que se deben aprovechar las posibilidades que nos brindan las tecnologías de la información. Los cambios metodológicos los podemos sistematizar en los siguientes puntos:

1. Cambio de mentalidad: Se debe producir un cambio de mentalidad tanto del profesor, que 
va a tener que organizar procesos de aprendizaje, dejando la clase magistral para momentos más reducidos, como para el alumno, que tendrá que asumir más responsabilidades, autonomía y compromiso. Es decir, se va a pasar de un aprendizaje basado en la enseñanza, cuyo eje es el profesor, a un modelo de enseñanza basado en el aprendizaje, centrado en el alumno y favoreciendo su implicación, actividad y protagonismo.

2. Cambio de roles: Va existir un cambio en el papel del profesor, que va a pasar de ser transmisor de información a facilitador y supervisor del aprendizaje. $Y$ lo que es más importante, también va a cambiar el papel del estudiante, pasando de ser espectador a actor, siendo parte activa en el proceso para lo que se va a exigir compromiso y responsabilidad, desarrollando especialmente un aprendizaje autónomo.

3. Actitudes básicas del alumno: Podemos decir que las tres siguientes son las actitudes básicas que el alumno deberá asumir: responsabilidad personal —debe ser el alumno quien asuma la responsabilidad de su propio aprendizaje-, autonomía —que el alumno actúe con iniciativa sin depender del profesor para todo-y colaboración - que el alumno vea como natural el trabajar en equipo-.

Evidentemente la aplicación de este nuevo paradigma lleva consigo una serie de inconvenientes que habrá que tener en cuenta. Entre ellos podemos citar el excesivo número de alumnos, la escasa disponibilidad de espacios, el escaso reconocimiento del profesor, los escasos recursos, etc. En cuanto a los cambios tecnológicos, en nuestro caso se centraron en la utilización de una plataforma creada por la propia universidad denominada Eudored.

\section{Diseño y planificación de la asignatura conforme al EEES}

A la hora de plantearse el diseño de la asignatura y su adaptación al modelo europeo seguimos el esquema que queda reflejado en la Figura 1. De tal manera que en primer lugar procederemos a identificar los objetivos que pretendemos conseguir y, en función de los mismos, dotaremos de contenido a nuestra asignatura. Una vez que sabemos lo que queremos conseguir y como lo vamos a lograr, procederemos a definir las actividades y recursos que vamos a utilizar para tal finalidad. En último lugar, estableceremos la evaluación a través de la especificación de los criterios que vamos a seguir; los instrumentos que vamos a emplear y los momentos en los que se va a producir tal evaluación.

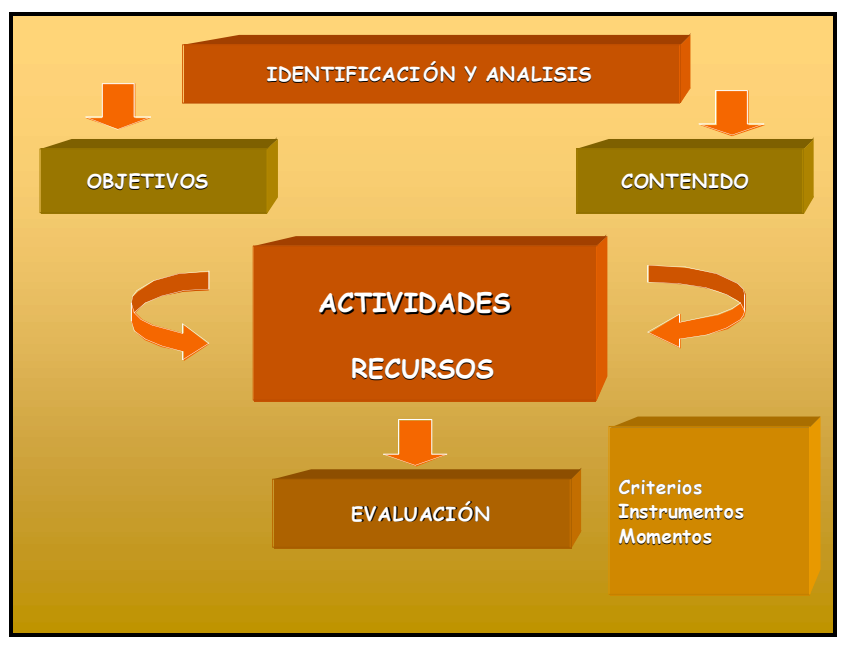

Figura 1. Esquema de planificación.

\subsection{Definición de objetivos}

Los objetivos pueden ser de dos tipos: generales, que son los más amplios y los utilizaremos como referencia de lo que queremos conseguir durante todo el curso; y específicos, aquéllos que plantearemos en cada bloque de contenido.

En su formulación nos debemos guiar por las siguientes premisas: deben ser claros, precisos, comprensibles por todos y fácilmente evaluables. Éstos no nos deben llevar solo a adquirir conceptos sino también habilidades, destrezas y actitudes. La tabla I muestra los objetivos generales que pretendemos conseguir a lo largo del curso en la asignatura de Catalogación.

\section{Objetivos generales}

La asignatura Catalogación descriptiva. I: Impresos modernos es de naturaleza eminentemente práctica y pretende capacitar a los/as alumnos/as para:

1. Realizar la descripción bibliográfica de monografías y publicaciones seriadas de acuerdo a las Reglas de catalogación (RC, 1999).

2. Elegir los puntos de acceso (principales y secundarios) de este tipo de publicaciones de acuerdo a las RC.

3. Redactar correctamente estos puntos de acceso (nombres de persona, de entidades y títulos uniformes).

4. Conocer las distintas metodologías existentes para describir partes componentes (distintos niveles de descripción, notas de contenido, catalogación analítica).

5. Realizar la catalogación de impresos modernos de los tipos anteriormente citados en formato MARC.

6. Conocer el funcionamiento del modulo de catalogación de diferentes sistemas de gestión de bibliotecas.

Tabla I. Objetivos generales de la asignatura. 


\subsection{Contenido}

A la hora de establecer el contenido de la asignatura tenemos que tener en cuenta tres ejes que nos van a guiar en la definición del mismo. Estos son: conceptos, habilidades y actitudes. Los conceptos nos llevan a aprender a conocer, es decir, comprender el mundo que nos rodea, desarrollando las capacidades profesionales pertinentes. Las habilidades nos permiten aprender a hacer, es decir, poner en práctica los conocimientos adquiridos. Finalmente, las actitudes nos facultan por un lado para aprender a convivir -nos dan el beneficio práctico del trabajo en común-, mientras que por otro nos capacitan para aprender a ser, es decir, nos otorgan un pensamiento autónomo y crítico.

En función de tales parámetros, la Tabla II nos muestra el contenido general de la asignatura objeto de estudio.

\begin{tabular}{ll}
\hline & \multicolumn{1}{c}{ Contenido } \\
\hline Bloque I & Introducción a la catalogación \\
\hline Bloque II & La descripción bibliográfica \\
\hline Bloque III & Redacción de los puntos de acceso \\
\hline Bloque IV & Elección de los puntos de acceso \\
\hline Bloque V & El formato MARC \\
\hline Bloque VI & Catalogación de publicaciones seriadas \\
\hline Bloque VII & Catalogación analítica \\
\hline Bloque VII & Módulo de catalogación SGBD \\
\hline
\end{tabular}

Tabla II. Contenido generales de la asignatura

Como podemos comprobar el contenido de la asignatura esta íntimamente ligado a la formulación de los objetivos.

\subsection{Diseño de actividades}

Cuando diseñamos una actividad la finalidad que debemos perseguir es la especificación de una serie de tareas que nos permitan conseguir los objetivos y desarrollar los contenidos previstos en el programa de la asignatura.

Podemos clasificar las actividades en presenciales y no presenciales. Presenciales serían todas aquellas que los alumnos realizan con la presencia del profesor. Dentro de esta categoría están: (1) teóricas —clase magistral, seminarios, presentación de trabajos individuales o en grupo etc.-, (2) prácticas - de aula (problemas), de campo con el profesor, etc.-, (3) otras -tutorías individuales o grupales, evaluaciones, etc. Sin embargo, ahora más que nunca en el EEES cobran importancia aquellas actividades de carácter no prensencial, aquellas que los alumnos realizan de forma autónoma, sin la intervención directa del profesor. Entre ellas podemos citar: (1) preparación de seminarios, lecturas, trabajos, memorias, investigaciones, etc.; (2) sesiones de trabajo en grupo no presencial; (3) prácticas de campo (visitas, visionado de películas, asistencias a conferencias impartidas por especialistas...); (4) estudio personal, en donde los alumnos preparan antes, completan e interiorizan después el contenido.

La tabla III muestra la definición de las actividades llevadas a cabo en el bloque $\mathrm{I}$.

\begin{tabular}{|c|c|c|}
\hline \multicolumn{3}{|c|}{ Objetivos, contenido y actividades para el Bloque I } \\
\hline Bloque I & \multicolumn{2}{|c|}{ Introducción a la catalogación } \\
\hline Objetivo & \multicolumn{2}{|c|}{$\begin{array}{l}\text { Introducir al alumno en el contexto de la } \\
\text { catalogación dentro del análisis } \\
\text { documental }\end{array}$} \\
\hline Contenido & \multicolumn{2}{|c|}{$\begin{array}{l}\text { 1. La catalogación dentro de la disciplina } \\
\text { del análisis documental. } \\
\text { 2. El producto del proceso de catalogar: } \\
\text { el catálogo. } \\
\text { 3. El contenido del catálogo: los registros } \\
\text { bibliográficos. }\end{array}$} \\
\hline \multirow{2}{*}{ Actividades } & Presenciales & Clases magistrales \\
\hline & No presenciales & Prácticas y visita \\
\hline
\end{tabular}

Tabla III. Especificación de las actividades para el Bloque I

\subsection{Evaluación}

El punto final del desarrollo de una asignatura es la evaluación de la misma para comprobar que el alumno ha alcanzado los objetivos previamente especificados y ha asimilado los contenidos. Como comentábamos anteriormente, debemos fijar previamente los criterios de evaluación. Éstos suelen definirse en la guía académica y están relacionados con los instrumentos que vamos a emplear para dicha evaluación. También debemos señalar los momentos en que se va a realizar dicha evaluación. La tabla IV muestra los criterios, instrumentos y momentos que hemos realizado evaluaciones.

\begin{tabular}{lll}
\hline \multicolumn{3}{c}{ Evaluación } \\
\hline Criterios & E. continua: $30 \%$ & E. final: $70 \%$ \\
\hline & - Exposición oral & \\
& - Presentación audiovisual \\
Instrumentos & - Redacción de informes \\
& - Realización del dossier \\
& - Examen escrito & \\
\hline \multirow{2}{*}{ Momentos } & Fin de bloque & $\begin{array}{l}\text { Fin de } \\
\text { asignatura }\end{array}$ \\
\hline
\end{tabular}

Tabla IV. Descripción de la evaluación 


\section{Conclusiones}

Una vez mostrada la metodología a seguir en cuanto a la planificación y adaptación de las asignaturas a las demandas del EEES, en este apartado vamos a destacar los puntos más relevantes a la hora de llevar a cabo tal tarea.

En primer lugar, diremos que hay dos conceptos claves a la hora de poner en marcha las nuevas materias, que son: el crédito ECTS y la guía académica. El primero es clave para cuantificar no sólo el trabajo del docente sino también del alumno, quien se convierte ahora en la pieza clave entorno al cual se estructura el método de aprendizaje. En segundo lugar, la guía académica se configura como una especie de contrato entre la institución universitaria y el estudiante, en dónde se específica claramente el programa de los planes de estudios a cursar de modo general, y de cada materia de manera particular.

El esquema a seguir a la hora de diseñar el desarrollo de la asignatura viene marcado por las siguientes pautas. En primer lugar debemos de realizar una identificación y análisis tanto de los objetivos que perseguimos como del contenido que debemos poner en marcha para cumplir los mismos. Identificados estos puntos, pasaremos a establecer las actividades y recursos a través de los cuales iremos desarrollando cada una de las materias, en función siempre de los objetivos y contenidos anteriormente especificados. Finalmente, llevaremos a cabo el proceso de evaluación.

Como conclusión fundamental del cambio que se opera entre los actuales planes y los planes adaptados al EEES diremos que, en primer lugar, se produce un cambio de paradigma que nos lleva de un aprendizaje basado en la enseñanza, cuyo eje es el profesor, a un modelo de enseñanza basado en el aprendizaje, centrado en el alumno, favoreciendo su implicación, actividad y protagonismo. Se produce a su vez un cambio de roles. Así, el profesor deja de ser transmisor de información y pasa a ser facilitador y supervisor del aprendizaje. Y cambia también el papel del estudiante, pasando de ser espectador a actor, siendo parte activa en el proceso, para lo que se le va a exigir compromiso y responsabilidad, desarrollando especialmente un aprendizaje autónomo.

\section{Referencias}

Abadal, E.; Miralpeix, C. (1998). La enseñanza de la Biblioteconomía y la Documentación en la Universidad española a finales de los noventa. // VI Jornadas Españolas de Documentación: Valencia, Oct., 1998. Valencia: FESABID, 1998, 29-47.
Bernal Aguado, J. L. (2006). Diseño curricular en la enseñanza universitaria desde la perspectiva de los ECTS. Zaragoza: Universidad de Zaragoza, 2006. ISBN 847791-227-0.

Bonete Perales, R. (2006). La adaptación de la Universidad de Salamanca al Espacio Europeo de Educación Superior: un desafía para todos. Salamanca: Universidad de Salamanca, Vicerrectorado de Planificación e Innovación Docentes, 2006.

García Marco, F. J., et. al. (2006). Diseño y coordinación curricular de las asignaturas de Tratamiento y Recuperación de la Información de la Diplomatura de Biblioteconomía y Documentación. Zaragoza: Prensa Universitarias de Zaragoza, 2006.

Frías Montoya, J. A. (1994). La formación universitaria de los catalogadores desde el punto de vista de los directores de las bibliotecas: avance de resultados. II IV Jornadas Españolas de Documentación Automatizada: Gijón, Oct., 1994. Barcelona: Parramón, 1994, 703-709.

Miguel, M. de (2006). Metodología de enseñanza y aprendizaje para el desarrollo de competencias: orientaciones para el profesorado universitario ante Espacio Europeo de Educación Superior. Madrid: Alianza Editorial, D.L. 2006.

Pinto Molina, M (2006). Prólogo. // García Marco, F. J. (aut.). Diseño y coordinación curricular de las asignaturas de Tratamiento y Recuperación de la Información de la Diplomatura de Biblioteconomía y Documentación. Zaragoza: Prensa Universitarias de Zaragoza, 2006, p. 7-8.

SFP (2005). Elaboración de la Guía Docente para la Convergencia Europea. València: Universitat de Valéncia, 2005.

Ubieto Artur, Isabel (1993). Los Planes de Estudio de las Diplomaturas en Biblioteconomía y Documentación en España (1978-1992). // Revista General de Información y Documentación. 3: 2 (1993) 143-165. 